\title{
Breast cancer and hormonal contraception: Should we rethink our concepts?
}

\section{CÂNCER de mama e CONTRACEPÇÃo hORMONAL: DEVEMOS REVER NOSSOS CONCEITOS?} \author{
Infância e Adolescência (Sogia Br) \\ Article received: $2 / 15 / 2018$ \\ Accepted for publication: 2/15/2018 \\ ${ }^{*}$ Correspondence: \\ bosco@fmj.br
}

JoÃo Bosco Ramos Borges ${ }^{1 *} \odot$, Renato Zocchio Torresan²

${ }^{1}$ Full Professor of Gynecology, Faculdade de Medicina de Jundiaí. President of the Sociedade Brasileira de Mastologia - SP (SBM SP). Secretary of the Associação Brasileira de Obstetrícia e Ginecologia da

${ }^{2} \mathrm{MD}$, PhD, Assistant Physician at the Centro de Atenção Integral à Saúde da Mulher (CAISM) da Universidade Estadual de Campinas (Unicamp). Vice-President of the SBM SP. President of the Comissão Nacional Especializada de Mastologia da Federação Brasileira das Associações de Ginecologia e Obstetrícia (Febrasgo)

The emergence and development of hormonal contraception was an achievement for the emancipation of women at the family, psychological and sexual levels, as well as social and professional, having reached great proportions in the 1960s with regard to the role of women in society, with multiple repercussions as it initiated and integrated the sexual revolution of the 1960s in the United States. Although its use has initially provoked some controversy in various areas of society, what is certain is that its utilization rate has been increasing steadily over these almost 60 years. To illustrate, oral contraceptives have a very high efficacy and more than 100,150,000 users worldwide. ${ }^{1}$

Currently, the goal is to offer women an increasingly safe and effective hormonal contraception. It is important to keep in mind that this type of drug does not only have a contraceptive effect. There are many inherent non-contraceptive benefits, such as protection against dysmenorrhea and menorrhagia, menstrual cycle irregularities, iron deficiency anemia, ectopic pregnancy, pelvic inflammatory disease, ovarian cysts, benign breast disease, and tumors of endometrium and ovary. But its great benefit is the decrease in the number of unwanted pregnancies and, particularly in Brazil, the reduction of unprotected abortion and its harmful consequences, including death.

Hormonal contraception can be administered by several routes, the most frequent being the oral route, but there are also injectable products, implants, vaginal rings, intrauterine progesterone devices and skin adhesives. Contraceptives may include combined estrogen and progesterone, progesterone alone, or different doses of ethinyl estradiol. The current trend is to gradually decrease the amount of estrogen in the pill. The first pills contained $150 \mu \mathrm{g}$ and we currently have pills with $15 \mu \mathrm{g} .{ }^{2}$
The association between use of hormonal contraception and breast cancer has been discussed for several years with results that are not always clear and conclusive.

In 2002, the CARE study, which compared 4,575 patients with oral contraceptives versus 4,682 patients in the control group, showed no difference in the risk of breast cancer. ${ }^{3}$

In 2008, the Wecare Study Group also failed to demonstrate any increase in breast cancer with the use of oral contraceptives. ${ }^{4}$

In 2006, an important meta-analysis that included 34 studies of premenopausal women conducted after 1980 and evaluated combined oral contraceptives (COCs) versus increased risk of premenopausal breast cancer showed a small risk for current COC use, increased risk for use before the first term pregnancy, and decreased risk for use after first term pregnancy. ${ }^{5}$

Another large study conducted in 2007 by the Royal College correlating COC and cancer risk (from 1968 to 2014) in a cohort of $23,796 \mathrm{COC}$ users compared to 23,377 non-users found RR for breast cancer of 0.98 (95CI 0.98-1.10). ${ }^{6}$

Studies conducted in the last $10-15$ years have failed to demonstrate increased breast cancer with the use of progestogen-treated intrauterine devices (IUDs). ${ }^{7,8}$

To date, no increased risk of breast cancer has been demonstrated with the use of hormonal contraception in patients with altered BRCA1 and BRCA2 genes. ${ }^{9}$

Regarding early breast cancer (women $<40$ years), in a case-control study conducted in the USA, Canada, and Australia $(n=1,073)$ in 2005, COC risk after 1975 yielded an odds ratio of 0.74 for non-carriers of mutation, OR 0.18 for BRCA1 and OR 0.92 for patients with BRCA2. Therefore, there was no evidence that the use of low-dose COCs increases the risk of early onset of breast cancer in patients with a 
mutation (there may even be a reduced risk for BRCA1 mutation carriers) and should not be contraindicated for a woman with germline mutation in BRCA1 or BRCA2. ${ }^{10}$

More recent studies also suggest that the associations between COC use and ovarian and breast cancer among women who carry BRCA1 or BRCA2 mutation are similar to those reported for the general population. ${ }^{11}$

Hannaford, in 2010, published a mortality risk assessment among women who used oral contraceptives compared to "never" users. The author observed that the absolute risk was $1 / 500$ in users under 35 years, increasing $24 \%$ in current users, $16 \% 4$ years after use, $7 \%$ after 5 years, and not significant after 10 years. The conclusion was that oral contraception was not associated with an increased risk of long-term death in this large UK cohort. According to the study, however, balance of risks and benefits may vary globally, depending on the patterns of oral contraception and risk of the disease. ${ }^{12}$

Another point of view is to evaluate the survival after breast cancer among previous users of COC. Women with invasive breast cancer participated in the Women's Contraceptive and Reproductive Experiences (CARE) study, cited above, a population-based case-control study $(4,565$ women aged 35-64 years), and the California Teachers Study (CTS) cohort with 3,929 women aged 28-91 years followed throughout life. There were 1,064 women who died in the CARE trial (mean follow-up, 8.6 years), while 523 died in the CTS (mean follow-up, 6.1 years). The authors concluded that the use of COC in the past is not associated with specific mortality from invasive breast cancer. These two independent studies demonstrated the non-association between COC use and survival among women with breast cancer. ${ }^{13}$

The recent result of the Royal College of General Practitioners Study on oral contraception with 44 years of follow-up was also positive. The longest study of the health effects of oral contraception in the world, published in 2017, aimed to examine the long-term risks or benefits of cancer associated with combined oral contraceptives, including overall survival. 46,022 women were recruited for up to 44 years. Rates of pathologies for users of combined oral contraceptives categorized as "EVER" (past users) and "NEVER" were calculated, along with standardized data for age, parity, social class and smoking. In total, 4,661 users (ever) were found with at least one cancer during 884,895 women-years of observation, and 2,341 non-users (never) with at least one cancer during 388,505 women-years of observation. The increased risk of breast and cervical cancer that has been observed in recent or current users appeared to be lost after approximately five years of interruption of the oral contraceptive with no evidence of recurrent cancer or increased risk in "ever" users over time. On the other hand, oral contraceptive use was associated with reduction in colorectal (incidence rate, 0.81 ), endometrial (incidence rate, 0.66 ) and ovarian cancer (incidence rate, 0.67), as well as lymphatic and hematopoietic (incidence rate, 0.74 ) cancer. There was no evidence of risk of developing a new cancer later in life for women who had used oral contraceptives. Thus, the overall calculation of cancer risk among past users of oral contraceptives was balanced, with the increased risk being offset by the benefits that persist for at least 30 years in relation to endometrial, ovarian and colorectal cancers. This leads to the conclusion that the majority of women who choose to use oral contraceptives do not expose themselves to a higher risk of cancer in the long run; on the contrary, with some types of cancer, many women benefit from significant risk reductions that persist for many years after discontinuation.

It should be kept in mind that contraceptives are used more often at younger ages and that breast cancer usually occurs much later, also considering that the associated risk for breast cancer is for recent or current user, disappearing after five years of discontinuation of the pill. ${ }^{14}$

However, different findings were also published in 2017 in a study that evaluated the associations between hormonal contraceptive use and the risk of invasive breast cancer in a nationwide prospective cohort including all women in Denmark aged 15-49 years age who had no cancer or venous thromboembolism and who did not receive treatment for infertility. There were 11,517 cases of breast cancer in a total of 1.8 million women who were followed on average for 10.9 years (a total of 19.6 million person-years). Compared with women who have never used hormonal contraception, the relative risk of breast cancer among all current and recent users of hormonal contraception was 1.20 (95CI 1.14-1.26). This risk increased from 1.09 (95CI 0.96-1.23) with less than one year of use to 1.38 (95CI 1.26-1.51) after more than 10 years of use $(\mathrm{p}=0.002)$.

And after discontinuing hormonal contraception, the risk of breast cancer was still higher among women who used hormonal contraceptives for five years or more compared to those who did not. Risk estimates associated with current or recent use of various oral contraceptives (estrogen-progestogen) ranged from 1.0 to 1.6.

The authors also concluded that women who currently or recently used a progestogen intrauterine device also had a higher risk of breast cancer compared to women who had never used hormonal contraceptives (RR 1.21; 95CI 1.11-1.33). 
The absolute increase in breast cancer diagnosed among current and recent users of any hormonal contraceptive was 13 cases (10 to 16 cases), with 95CI, per 100,000 people, or approximately one extra case of breast cancer per 7,690 women using hormonal contraception for one year. ${ }^{15}$

Commenting on this study, I now make the observations of Febrasgo and its National Commission on Mastology. There is an increase in risk ( 1.3 new cases per 100,000 women), a risk that increased with time of use, and no increase in risk for users with less than 5 years. And for women under 35 years of age, who represent the vast majority of users of hormonal contraceptives, the study showed an increase of 0.2 cases of breast cancer in every 10,000 women per year. Remember that these data should be brought to light in view of the great benefit of hormonal contraception in the female reproductive context.

If we analyze the greatest increase in risk in the group of women aged over 35 or 40 years, this can be misinterpreted in view of the natural history of breast cancer, which has a higher incidence in this age group, although the comparative analysis against the non-hormonal contraceptive users has shown a relative increase in risk.

The increased risk for breast cancer among users of combined hormonal contraceptives has disappeared after discontinuation of the contraceptive, and this finding may demonstrate the non-influence of contraceptives on the genesis of breast cancer. If so, the risk would remain high all the time after discontinuation. Thus, there is no evidence of a causal relationship between contraceptive use and risk of breast cancer.

It should be considered that this study did not evaluate breast cancer mortality in users of hormonal contraceptives and therefore it is not possible to infer from the data presented that the increased risk described has the potential to aggravate the prognosis of breast cancer associated with use of hormonal contraceptives. These women are usually kept under increased surveillance and, as with hormone therapy users, the disease-specific mortality may be even lower when stratified by staging at diagnosis. Because breast cancer is a multifactorial disease, the sum of risks is more important than the increased risk conferred by any individual factor, that is, obesity, nulliparity, sedentary lifestyle, history of previous proliferative disease and family history.

Finally, it should be remembered that hormonal contraceptives represent a long-studied pharmacological class and are associated with high safety. The risk-benefit anal- ysis should be individualized, and the final clinical decision should be elaborated after discussion based on the WHO eligibility criteria for the use of contraceptives.

Again, it is necessary to particularize the cases and the discussion with each patient to substantiate the indication of hormonal contraception, always considering the enormous known benefits. In addition to the drastic reduction of unwanted pregnancies, there is a decreased risk of ovarian, endometrial and rectal cancer and the treatment of numerous hormonal dysfunctions and abnormal bleeding.

\section{Conflict of interest}

The authors declare no conflict of interest.

\section{REFERENCES}

1. United Nations Department of Economic and Social Affairs Population Division. World contraceptive patterns 2013. New York: United Nations; 2013

2. Poli MEH, Mello CR, Machado RB, Pinho Neto JS, Spinola PG, Tomas GS, et al. Manual de anticoncepção da FEBRASGO. Femina. 2009; 37(9):459-62.

3. Marchbanks PA, McDonald JA, Wilson HG, Folger SG, Mandel MG, Daling JR, et al. Oral contraceptives and the risk of breast cancer. N Engl J Med. 2002; 346(26):2025-32.

4. Figueiredo JC, Bernstein L, Capanu M, Malone KE, Lynch CF, Anton-Culver $\mathrm{H}$, et al.; WECARE Study Group. Oral contraceptives, postmenopausal hormone and risk of asynchronous bilateral breast cancer: The Wecare Study Group. J Clin Oncol. 2008; 26(9):1411-8.

5. Kahlenborn C, Modugno F, Potter DM, Severs WB. Oral contraceptive use as a risk factor for premenopausal breast cancer: a meta-analysis. Mayo Clin Proc. 2006; 81(10):1290-302.

6. Hannaford PC, Selvaraj S, Elliott AM, Angus V, Iversen L, Lee AJ. Cancer risk among users of oral contraceptives: cohort data from the Royal College of General Practitioner's oral contraception study. BMJ. 2007; 335(7621):651.

7. Curtis KM, Marchbanks PA, Peterson HB. Neoplasia with use of intrauterine devices. Contraception. 2008; 77(2):138.

8. Backman T, Rauramo I, Jaakkola K, Inki P, Vaahtera K, Launonen A, Koskenvuo M. Use of the levonorgestrel-releasing intrauterine system and breast cancer. Obstet Gynecol. 2005; 106(4):813-7.

9. Grenader T, Peretz T, Lifchitz M, Shavit L. BRCA1 and BRCA2 germ line mutations and oral contraceptives: to use or not to use. Breast. 2005; 14(4):264-8.

10. Milne RL, Knight JA, John EM, Dite GS, Balbuena R, Ziogas A, et al. Oral contraceptive use and risk of early-onset breast cancer in carriers and noncarriers of BRCA1 and BRCA2 mutations. Cancer Epidemiol Biomarkers Prev. 2005; 14(2):350-6.

11. Moorman PG, Havrilesky LJ, Gierisch JM, Coeytaux RR, Lowery WJ, Peragallo Urrutia R, et al. Oral contraceptives and risk of ovarian cancer and breast cancer among high-risk women: a systematic review and meta-analysis. J Clin Oncol. 2013; 31(33):4188-98.

12. Hannaford PC, Iversen L, Macfarlane TV, Elliott AM, Angus V, Lee AJ. Mortality among contraceptive pill users: cohort evidence from Royal College of General Practitioners' Oral Contraception Study. BMJ. 2010; 340:c927.

13. Lu Y, Ma H, Malone KE, Norman SA, Sullivan-Halley J, Strom BL, et al. Oral contraceptive use and survival in women with invasive breast cancer. Cancer Epidemiol Biomarkers Prev. 2011; 20(7):1391-7.

14. Iversen L, Sivasubramaniam S, Lee AJ, Fielding S, Hannaford PC. Lifetime cancer risk and combined oral contraceptives: the Royal College of General Practitioners' Oral Contraception Study. Am J Obstet Gynecol. 2017; 216(6):580.e1-580.e9.

15. Mørch LS, Skovlund CW, Hannaford PC, Iversen L, Fielding S, Lidegaard $\varnothing$. Contemporary hormonal contraception and the risk of breast cancer. $\mathrm{N}$ Engl J Med. 2017; 377:2228-39. 